\title{
Terminological and Metrological Aspects in Siepser-Type Iris Suture Procedures
}

\section{Bordeianu CD*}

Private Practice, Ploiesti, Prahova, Romania

*Corresponding author: Bordeianu ConstantinDan, 15, Cameliei st., Bl. 26, sc. B, et. I, Ap. 26, Ploiesti, Romania

Received: March 29, 2021; Accepted: April 14, 2021; Published: April 21, 2021

\begin{abstract}
Instead of contemporary plethora, with tenths of different terms for only 7 elements, I suggest a system in which the main criterion is the length of the 2 filament ends just before the loop is externalized: $\boldsymbol{L}$ : long end, and $\mathbf{S}$ : short end. The name of the 2 corneal pathways, and 2 iris bites will rely on the name of the corresponding filament end that passes through, just before the loop is externalized: Sp/SP: short-end puncture/paracentesis, Lp/LP: long-end puncture/paracentesis, SIB: short-end iris bite, LIB: long-end iris bite. When the loop is externalized, the "intermediary segment": $(I)$ is created from $L$. The limits of (I) have been adjusted: instead of generally admitted limits from the middistance between SIB and LIB, to the middle of the externalized loop, I suggest that the $S /(I)$ limit be situated at $1 \mathrm{~mm}$ after the exit point of the loop from $S P$, and the $(I) / L$ limit be situated at the re-entry point of the loop in $S P$.
\end{abstract}

The suggested terminology and limits of $(I)$ allow a clear and unequivocal description of techniques, simplify the repartition of functions per filament segment, avoid misunderstanding as cause of failure, facilitate the establishment of rules for success valid in any Siepser-type suture, and allow a new systematization of all iris suture procedures in a structure with a common stem, 2 branches (McCannel, Siepser) and leaves represented by technical variants.

Keywords: Siepser knot; New terms; New segment limits; Rules for success; Isometric manner of performing endocular maneuvers

\section{Abbreviation}

\section{Classical terms}

C: Caudal End; F: Frontal End; (I): Iris Segment; fib: First Iris Bite; lib: Last Iris Bite; np: Entry Puncture; nP: Entry Paracentesis; xp: Exit Puncture; xP: Exit Paracentesis; f: Angled Forceps

\section{Suggested terms}

S: Short End; L: Long End; (I): Intermediary Segment; SIB: Short End Iris Bite; LIB: Long End Iris Bite; $\mathbf{S p}$ : Short End Puncture; SP: Short End Paracentesis; $L \boldsymbol{p}$ : Long End Puncture; LP: Long End Paracentesis

\section{Introduction}

After 1994, when Siepser [1] suggested the slip knot, the iris suture tended to become popular, either to close iris wounds, to cover iris defects, to treat paralytic mydriasis, to achieve PC IOL fixation when the capsular bag is absent, or to induce firm iris/capsule synechia when the bag/IOL complex is displaced because of localized zonule absence, or of its general weakness. The last purpose is achieved either directly - by capsule/iris suture [2,3], or indirectly - by a suture between the iris and an implanted capsular tension ring [4,4'].

In any iris suture (Figure 1A), the frontal end attached to the needle passes through the entry corneal pathway, and the first iris bite; performs the suture purpose, takes the last iris bite, and gets out through the exit corneal pathway. When the entry pathway is a paracentesis, the needle must advance with lateral movements, to avoid nailing stromal strands from the cornea. When the exit pathway is a paracentesis, a "guiding cannula" [5] will avoid both the endothelial trauma while searching the internal opening, and the accidental corneal stroma bites.

\section{The Siepser knot is based on Siepser's principle}

Bringing in the same paracentesis the 2 filament segments that will be knotted, and the segment that will drag the loose knot over the iris defect (Figure 1B), where it will be tightened without stretching the iris.

\section{I named this manner of knot tightening the "isometric" manner}

Without changing the position of the element being worked on. I used this term first in a paper about phaco-technique [6], in a period of time when the probe pushed the nucleus so much that it disappeared from the screen. I consider it to be superior to ulterior terms like "no motion, slow motion or immobile movement phacoemulsification" [7]. Now, I think that it's use can be extended to all intraocular maneuvers.

\section{The Siepser principle is put in practice by Siepser maneuver}

Nail the filament between the last iris bite and exit puncture and externalize the loop through entry puncture.

Several variants for knot construction exist in literature: Siepser [1], throws the caudal end around iris segment (Figure 2A), and pulls both ends in opposite directions. A similar sequence closes the "granny's" knot. Osher [8] has modified the maneuver by alternating the trailing end entry into the loop from above and from below in the 

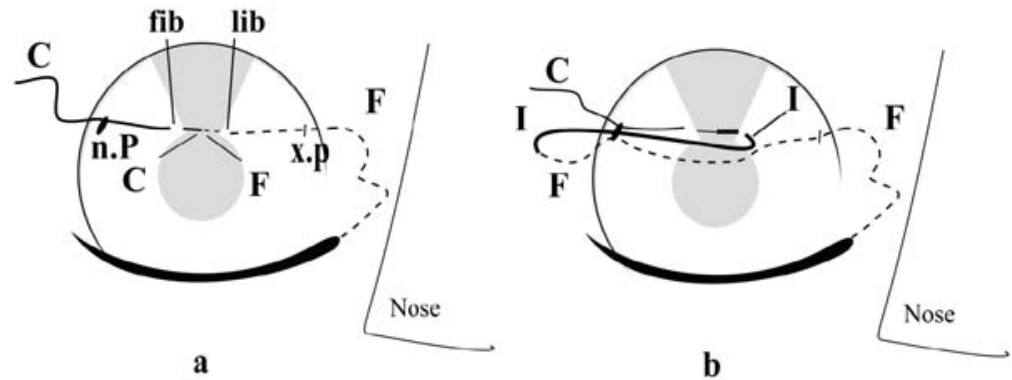

Figure 1: Schematic representation of filament path in Siepser type sutures, right eye, classical terms, needle passage right-left: a) Before loop externalization; b) After loop externalization.

2-3 sequences of knot formation, and the result was a real "square knot". Condon [9] (Figure 2B) and Ahmed [10] (Figure 2C) eased the throwing maneuvers by making coils around the tying forceps in one filament segment, and by pulling the other end through these coils. Yao and Jhanji [11] (Figure 2D) replaced the tying forceps with the long needle mounted on the frontal end. All these variants suppose 2-3 loop externalizations, and 2-3 successive knots: the first - for lips juxtaposition, and the other/s - for knot closure. Narang and Agarwall [12] suggested the SFT (single pass, four throws) knot, in which the closure knot lacks. Finally, Schoenberg [13] imagined a particular needle path so that the cut ends of the knot be oriented toward IOL, sparing the endothelium.

\section{Terminological Aspects}

The learning curve of the Siepser knot is long enough, with failed attempts, partly because the filament is thin and pale tinted, partly because the procedure description does not benefit from a unified terminology that could allow the formulation of rules for success valid in any situation. Although we need proper terms for only 7 structures ( 3 filament segments, 2 corneal pathways, and 2 iris bites), an excessive terminological variability exists, and this explains the misunderstanding and failure.

1. Speaking of terminological variability, the ends of suture filament are named either frontal/caudal, leading/trailing, nasal/ temporal, left/right, distal/proximal, initial/terminal, inferior/ superior, your end/loose end, mounted on needle/free end, one end/ the other end, first end/the second end, strand No 1/strand No 3, or a combination of these terms $[1,8-20]$. On the right eye, the right handed surgeon will usually pass the needle from the right to the left, so that the frontal end is in the same time nasal, leading, left, distal, initial, first, superior, loose end, mounted on the needle, one end, or strand No 1; whereas the caudal end is the trailing, temporal, right, proximal, terminal, second, inferior, your end, free end, the other end, or strand No 3.

Entering into terminological details, the "frontal/caudal", "leading/trailing", "initial/ terminal", "one/another", "first/second", and "strand no1/strand no3" pairs of terms are apparently neutral, and their use may seem logical, but: when the knot construction site is reversed, the functions change; when the needle passage direction is changed, the names are reversed, and the difficulty remains. The "free/mounted on the needle end" doesn't work in cases in which the needled end must be cut short: in this case this end cannot be named "mounted on the needle." The meaning of the "nasal/temporal" pair of terms changes with the patient eye's laterality. As for the "right left" pair of terms, other questions may confuse the beginner, such as: "Whose laterality? Patients' or surgeons'? If it's the surgeon's, then in which position is he placed? Temporal, or at 12 o'clock?" The suture orientation brings other difficulties, as the suture may be parallel to $0-180^{\circ}$ meridian, to $90-270^{\circ}$, or may be oblique. For a surgeon placed at $90^{\circ}$, when the needle passes parallel to $0-180^{\circ}$ meridian, there is no frontal-caudal, inferior-superior, distal-proximal, or your end-loose end. For a doctor placed temporally, a suture parallel to the $90-270^{\circ}$ meridian will not have right-left, distal/proximal, temporal/nasal, or your end/loose end. The Siepser maneuver brings an additional difficulty, because the 3 thin and poorly tinted filament segments crowded in a tiny puncture - are difficult to be observed, and may be easily confused.

2. When we pass to the names of corneal pathways, and iris bites, we find other causes of confusion induced by the existing pairs

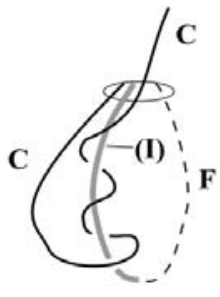

a

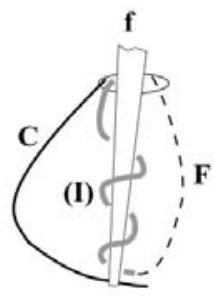

b

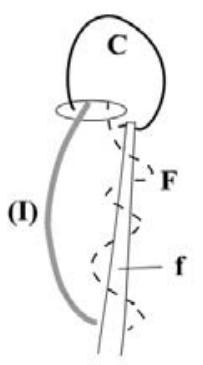

c

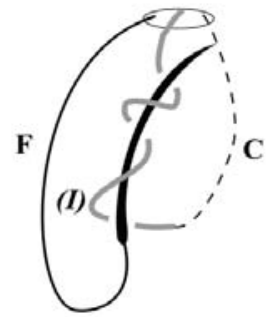

d

Figure 2: Technical variants in Siepser knot construction, classical terms: a) Siepser [1]; b) Condon [9]; c) Ahmed [10]; d) Yao and Jhanji [11]. 
of terms [1,8-20]: "entry/exit, first/second, left/right, nasal/temporal, frontal/caudal, distal/proximal, initial/terminal, one/another, first/last, inferior/superior, or a combination of these terms. The observations about the name of filament ends remain valid for the name of iris bites and corneal pathways. The corneal pathway might be a puncture or a paracentesis": in some situations the needle may enter and/or exit the anterior chamber directly through the peripheral cornea, through simple punctures; other situations need paracentesis, incisions made before needle passage.

3. The terminological variability slightly affects the median segment of the filament, between the 2 ends: most authors name it the "iris segment" $[1,8,9,11-20]$; only one author names it "the strand No 2" [10]. All authors agree that it ends in the middle of the externalized loop. Taking this as an example, we may consider that it begins in the middle of the space between the first and the last iris bites (Figure 1B).

4. The manner in which these terms are used in procedure description brings other causes of confusion, because too many variables intervene. For concision, I replaced the existing names with symbols: F - for "the frontal end"; C - for "the caudal end", (I) - for "iris segment", np - for "the entry puncture", nP - for "the entry paracentesis", $\mathbf{x p}$ - for "the exit puncture", $\mathbf{x P}$ - for "the exit paracentesis", and fib/lib - for "the first iris bite" or "the last iris bite". All symbols are written with bold characters, to differentiate them from the symbols of the suggested, new names, that will be written with bold, italic, underlined characters. I placed the symbol for "iris segment" between round brackets (I), to differentiate it from the personal pronoun, first person, singular.

a) Usually, the right handed surgeon will pass the needle from right to left (Figure 3A). The entry approach must be a paracentesis, to allow the intraocular maneuvers; whereas the exit approach may be a puncture. The $\mathbf{C}$ is on surgeon's right, passes through $\mathbf{n P}$, and fib, and is short, to ease the throwing maneuver. The $\mathbf{F}$ is on the left, after passing through lib, and xp. It maintains the needle, and must remain long, to allow the loop formation. The knot construction site is on the right side of the surgeon, close to the $\mathbf{n P}$. The loop is formed by nailing $\mathbf{F}$ between lib and $\mathbf{x p}$, and is externalized through $\mathbf{n P}$. The knot is made either by throwing $\mathbf{C}$ around (I) $[1,8,12,13]$ or by coiling around a forceps $[9,10]$ either (I) [9] or $\mathbf{F}[10]$ and by pulling C through these coils.

When we analyze the throws' progression, no author addressed this aspect. In Siepser-Osher type variants $[1,8,12,13]$, anyone can see the throws progress toward lib. However, nobody stated this as a rule, and thus it may pass unobserved, with possible failures. The use of instruments complicates the situation, because now there are no throws of $\mathbf{C}$, but coils are made around the forceps in other filament segments. The only transmitted information is that the forceps must be held over (I) [9] or over F [10], with the tip oriented toward loop apex [9] or loop base [10]. The coils are made in (I) [9] or in $\mathbf{F}$ [10], and the pulled segment through the coils is the $\mathbf{C}[9,10]$ or $\mathbf{F}[11]$

b) Sometimes, in the examples above, after the filament has been passed, local conditions impose to build the knot close to the exit approach. In this case, the following modifications must be done: the exit approach must be transformed into a paracentesis, a difficult maneuver when the filament exists inside the puncture (the helping

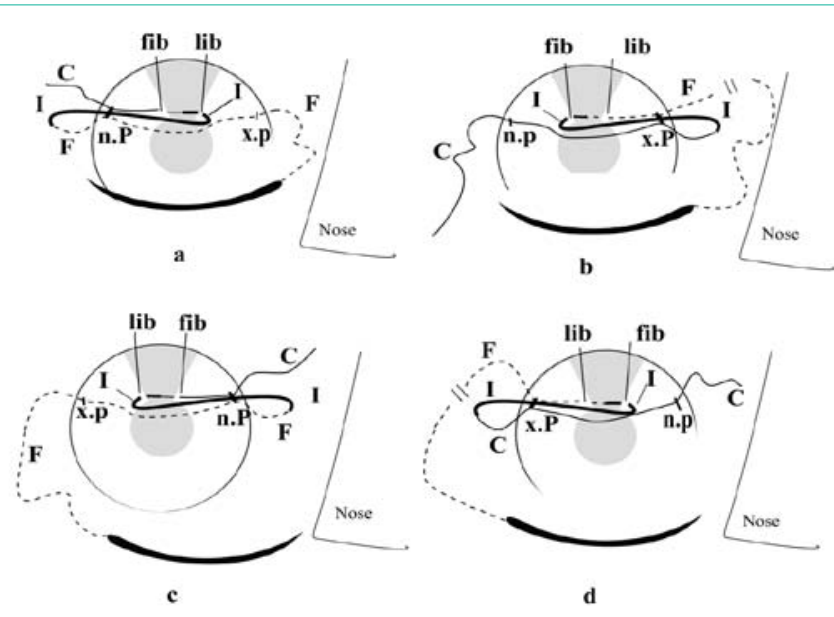

Figure 3: The 4 execution variants in Siepser maneuver, classical terms, right eye: a) Right handed surgeon, needle passage right-left; knot construction site close to $\mathbf{n P}$; b) Right handed surgeon, needle passage right-left, knot construction site close to $\mathbf{X P}$; c) Left handed surgeon, needle passage leftright, knot construction site close to $\mathbf{n P}$; d) Left handed surgeon, needle passage left-right, knot construction site close to $\mathbf{X P}$.

maneuver is detailed at page 5 , paragraph c). The $\mathbf{C}$ end remains long, on the right. The $\mathbf{F}$ end remains short, on the left, after cutting off the needle. The loop is formed by nailing $\mathbf{C}$ between $\mathbf{n P}$ and $\mathbf{f i b}$, and is externalized through $\mathbf{x P}$ (Figure $3 \mathrm{~B}$ ). The knotting maneuver consists of throwing the shortened $\mathbf{F}$ around (I) $[1,8,12,13]$ or in coiling around a forceps either (I) [9] or $\mathbf{C}$ [10], and in pulling $\mathbf{F}$ through these coils (opposite to what happened in the previous example).

c) When surgeon's hand dominance, or local configuration imposes the needle passage from the left to the right, usually the knot is made close to entry approach. In this case the second scenario will be applied, with some peculiarities: the entry approach must be a paracentesis: $\mathbf{n P}$. The exit approach remains a puncture: $\mathbf{x p}$. The $\mathbf{F}$ will be on the right, will be long, and will keep the needle, while $\mathbf{C}$ will be on the left, will be short, and will be thrown (Figure 3C).

d) When in this situation, the local conditions impose the knot construction close to the exit approach, the first scenario will be applied, with particularities: the $\mathbf{x p}$ must be transformed into $\mathbf{x P}$ (see the helping maneuver at page 5, paragraph $\mathrm{c}$ ), the $\mathbf{F}$ will be on the right, will be cut short, will lose the needle, and will be thrown, while C will remain long, on the left (Figure 3D).

For the left eye, other 4 figures will give different names for same of these elements.

$\boldsymbol{e}$ / In variants $\mathrm{b} /$ and $\mathrm{d} /$ (Figure 3B, and 3D), it is also possible to use the long needle as coiling tool [11] (Figure 2D), with the following peculiarities: $\mathbf{F}$ must remain needled and short, by being pulled less through $\mathbf{x P}$. The needle must be held above (I) like the forceps in Condon variant [9], but its tip must be oriented toward loop base, not toward loop apex, and the segment that is pulled through the coils is F, not $\mathbf{C}$. This variant cannot be used in the cases shown in Figure 3A and $3 \mathrm{C}$, because the needled end gets out through $\mathbf{x p}$, while the loop is externalized through $\mathbf{n P}$.

To resume, the terminologic variability is determined by the patient's eye laterality, by the surgeon's predominant hand, by the 

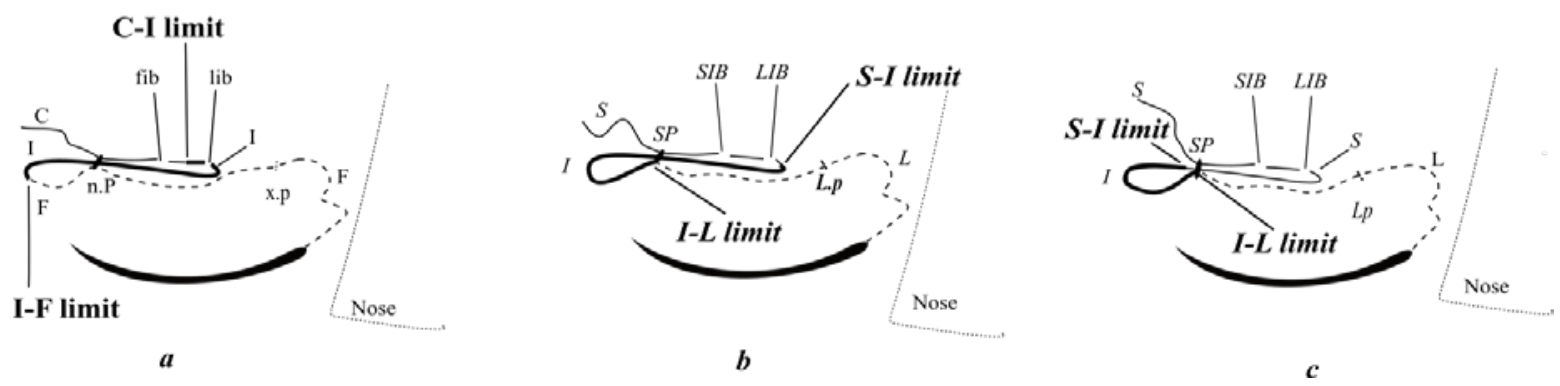

Figure 4: The suggested limits of (I): a) The existing situation; b) The first variant; c) The final variant.

used instrument and by the used terminology. It will affect the name of the segments, the selection of the segment that must be thrown, the selection of the segment around which the throws are done, the direction in which the throws progress, and how the throws are done (with or without instruments). The knot construction site is usually close to the entry pathway. If local conditions make the knot construction easier close to the exit pathway, the names of the filament segments remain, but the actions fulfilled by each end will be reversed. When the passage is easier from the left to the right (in case of left handed surgeon, or left eye), some names will be reversed, but the functions remain. Finally, when in the situation above local conditions make the knot construction easier close to the exit pathway, both the names and the function's laterality will be reversed compared with the situation in Figure 3A. When instruments are used, the variability affects both the used instrument, and how the instruments are used. The cherry on the cake is put by some authors who do not specify the segment around which the throws are done $[11,12]$, and by all authors, who do not mention the direction in which the throws must progress to ensure success.

The resulting imbroglio forbids the formulation of valid rules for success valid in any situation because the road to success is paved with details: sometimes the details differ according to the local configuration and to the surgeon's predominant hand; some other times, they completely lack. In such confusing conditions, with so many variables, the surgical stress may confuse even experienced surgeons.

\section{Metrological Aspects}

The classical limits of (I), from the middle of the space between fib and lib, to the middle of the externalized loop (Figure 4A) bring other difficulties.

a) The old limits of (I) make difficult the understanding of different variants in Siepser technique. It is certain that the contact between fib and lib is induced by a crossed pull of the knot on 2 different segments: $\mathbf{C}$ pulls fib in one direction, while (I) pulls lib in opposite direction. However, before pulling, the knot must be constructed, and the used maneuvers are a matter of choice: the externalized loop may be created through $\mathbf{n P}$ or $\mathbf{x P}$, by nailing $\mathbf{F}$ between lib and $\mathbf{x p}$, or $\mathbf{C}$ between $\mathbf{n p}$ and $\mathbf{f i b}$; the knot may be formed by throwing $\mathbf{C}$, or $\mathbf{F}$ around (I); the throws may begin from above or from below the loop, and may advance toward $\mathbf{F}$, or toward $\mathbf{C}$. The use of instruments brings other variables. With so many variables, the choice of the right variant may be difficult, and this is important, because some variants produce failure. b) The repartition of functions per filament segment lacks constancy. Usually, $\mathbf{C}$ has 3 functions: it takes fib, performs the throws, and tightens the knot; the (I) has 2 functions (it takes lib, and is the element around which the throws are made); in almost all variants $\mathbf{F}$ has 2 functions (it drags the knot over the iris defect, and tightens it). But in one variant, $\mathbf{F}$ is the element around which the throws are made [10], and in other variant it is the element that is drawn through the coils [11]. As a consequence, (I) may have 2-3 functions, while $\mathbf{F}$ may have 2-4.

c) The old limits of (I) complicate the classification of Siepser technique variants by granting significance to the differences between 2 variants that use instruments. In one variant (Figure 2B) [9], the angled forceps used for making the coils is held over (I), and in other (Figure 2C) [10] - over F. In one variant [9] the forceps tip is oriented toward the loop apex, in the other [10] - toward the loop base. In one variant [9] the coils are made in (I), in the other [10] - in F. In one variant the coils seem to progress toward the loop apex [9], and in the other - toward the loop base [10]. In one variant $\mathbf{C}$ makes the turns around (I) [9], and in the other - around $\mathbf{F}$ [10]. There are enough differences to separate these variants. We will see that the new limits of (I) simplify everything.

d) With this high variability in terms and segments limits it is impossible to elaborate general rules for success valid in any particular situation.

e) Finally, with the existing segments limits, no connection can be made between McCannel and Siepser type procedures, although both are used for the same purpose.

I must underline that all the discussed examples seem complicated even if I used one single set of terms. I wonder how complicated would the explanation be if I used a combination of more than 12 existing sets of terms? In order to unify the terminology, to avoid errors caused by misunderstanding, to shorten the learning curve, and to ease the formulation of general rules for success valid in any situation, my paper suggests new terms, and new limits for (I).

\section{Terminological Suggestions}

\section{The new terms must maintain their significance in any situation}

For that they must be connected with a certain characteristic valid in any situation. This characteristic is the length of each filament end after the passage through iris, just before the loop is externalized. In any situation, one end must be long - to allow the creation of the median segment, whereas the other end must be short - to ease the 

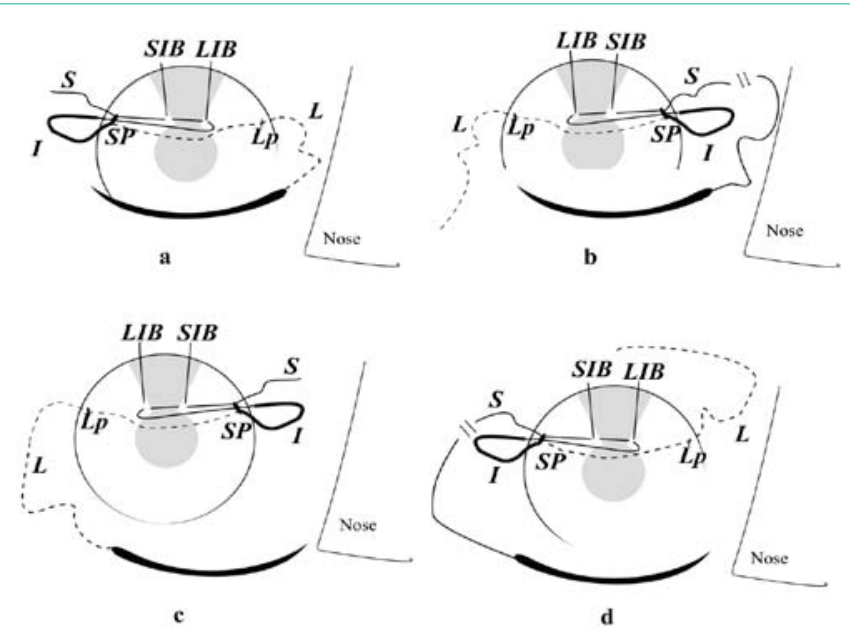

Figure 5: New terms, execution variants in Siepser-type maneuver, right eye: a) Right handed surgeon, needle passage right-left; knot construction site close to SP; b) Right handed surgeon, needle passage right-left, knot construction site close to LP; c) Left handed surgeon, needle passage leftright, knot construction site close to SP; d) Left handed surgeon, needle passage left-right, knot construction site close to LP.

throwing maneuvers.

a) As a consequence, there will always exist a "long end" $L$ and a "short end" $\mathbf{S}$, no matter the direction in which the needle is passed, and where the knot construction site is situated. The punctures and the iris bites will be named after the name of the filament that passes through, exactly before loop externalization: "long-end puncture/ paracentesis": $\mathbf{L p} / \mathbf{L P}$, and "short-end puncture/paracentesis": $\mathbf{S p /}$ SP; "long-end iris bite": LIB, and "short-end iris bite": SIB. All suggested symbols are written with bold, italic, underlined characters, to ease the differentiation between the old and the new terms.

After all these are set, when the loop is externalized, the median segment is created from $\boldsymbol{L}$. I intended to maintain (I) as symbol, a vowel that will allow mnemonic formulas both with the old and with the new symbols of filament ends $(F, C ; S, L)$. As the Figure 4C proves that the segment has lost any contact with iris, the name "iris segment" was no longer fit, and I named it "intermediary segment", with (I) as symbol, that maintains the round brackets to differentiate it from the personal pronoun, first person, singular.

b) The moment when each segment gets its name is situated just before nailing $\boldsymbol{L}$ for loop creation. However, the decision regarding the knot construction site, may change till the last moment. Figure $5 \mathrm{~A}-5 \mathrm{D}$ shows all 4 possible variants for the right eye, with the new terms and new limits of (I). A comparison with Figure 3A-3D shows evidence that the new terminology simplifies everything, because in all 4 examples only $\boldsymbol{L}$ will be nailed, this will always happen between LIB and $\boldsymbol{L P}$, and the loop will be externalized only through $\boldsymbol{S P}$. Thus, with the new terms, there is no need of 4 figures for the right eye and 4 figures for the left eye: one figure is enough for all situations.

c) Sometimes, the conditions are at their limits, and local difficulties may impose a last moment change of the knot construction site, even after shortening the needled end. The length of the filament ends is easy to adjust: simply pull the shortened former $L$, to make it long again. On the contrary, the transformation of a puncture into a paracentesis is extremely difficult when a filament already exists inside the puncture: the filament may be accidentally cut, or some stromal strands may remain between the puncture and the paracentesis pathways. In both situations, the filament must be ablated and replaced, with new iris trauma. I avoided this by making the new paracentesis near the puncture and by nailing or grasping the filament within the anterior chamber, and bringing it out from puncture to paracentesis. However, the simplest and safest solution when the selection of knot construction site may pose problems, is to make both corneal pathways - paracentesis from the beginning, and to postpone the naming decision until all doubts have been eliminated.

\section{Metrological Suggestions}

The disadvantages of the old, existing limits of (I) discussed at page 4 would be avoided if these limits would be modified in a manner that would not influence the practical execution of any maneuver, in any variant, but would improve everything at the conceptual level.

Compared with the existing situation (Figure 4A), the first variant (Figure 4B), displaced the former $\mathbf{C} /(\mathbf{I})$ limit from the middle of fib/ lib space to $1 \mathrm{~mm}$ after the exit from $\mathbf{L} \boldsymbol{B}$, so that the suggested $\mathbf{S}$ be able to fulfill alone the suture purpose (approaching the iris bites), unlike the generally admitted situation when the suture purpose is executed by opposite traction of 2 separate segments [C and (I)]. In the same time, I moved the (I)/F limit to the end of externalized loop, in order to annul the generally admitted situation, in which the loop is formed by 2 different segments, (I) and F, so that there exist variants that make coils in (I) $[9,11]$, or in $\mathbf{F}$ [10]; variants that pull through these coils either $\mathbf{C}[9,10]$, or $\mathbf{F}[11]$. Now, all variants throw the new $S$ around the new $(I)$, or achieve the same by making coils in (I), and by pulling $S$ through those coils.

The last modification affects the $\boldsymbol{S} /(\boldsymbol{I})$ limit (Figure 4C) by further moving it up to $1 \mathrm{~mm}$ after the exit point of $\boldsymbol{S}$ from $\mathbf{S P}(1 \mathrm{~mm}$ after the beginning of the externalized loop), because the intracameral segment of (I) had no practical meaning, and because with this modification, the new $\boldsymbol{S}$ in Siepser-type procedures will reproduce the entire filament path in Mc Cannel [21] - type ones. For that, the new $\boldsymbol{S}$ had to be prolonged up to $1 \mathrm{~mm}$ after the exit point from $\boldsymbol{S P}$, and be formed by the old $\mathbf{C}+$ the intracameral segment of the old (I) + the segment crossing $\mathbf{S P}+1 \mathrm{~mm}$ after the exit point. With this modification, it is possible to unify all variants of iris suture in one single scheme, with a common stem, 2 vigorous branches (McCannel and Siepser), and leaves - represented by the each technical variant. This scheme will be detailed after describing the new personal variants both of the McCannel type and of Siepser type of iris suture.

I also affirmed that the new limits of (I) will simplify the classification of Siepser-type variants. The old limits of (I) complicate the classification by allowing the separation of variants that throw $\mathbf{C}$ around (I), or around $\mathbf{F}$, of variants that pull $\mathbf{C}$ through the coils made in (I) or in $\mathbf{F}$ (Figure 2A, 2B, 2C), and of variants in which $\mathbf{C}$ is needless, or needled (Figure 2D). Unlike this situation, with the new terminology and limits of (I), if, at the end of knot construction, we will use imagination and straighten the coiled segment (Figure 6B, 6C, 6D), we will observe that in all variants $S$ makes throws around (I), like in Figure $2 \mathrm{~A}$ and $6 \mathrm{~A}$. This proves that no matter how the knot is constructed (by throwing $\mathbf{S}$ around other filament segment, or by pulling $\boldsymbol{S}$ through coils made in other filament segments), the 

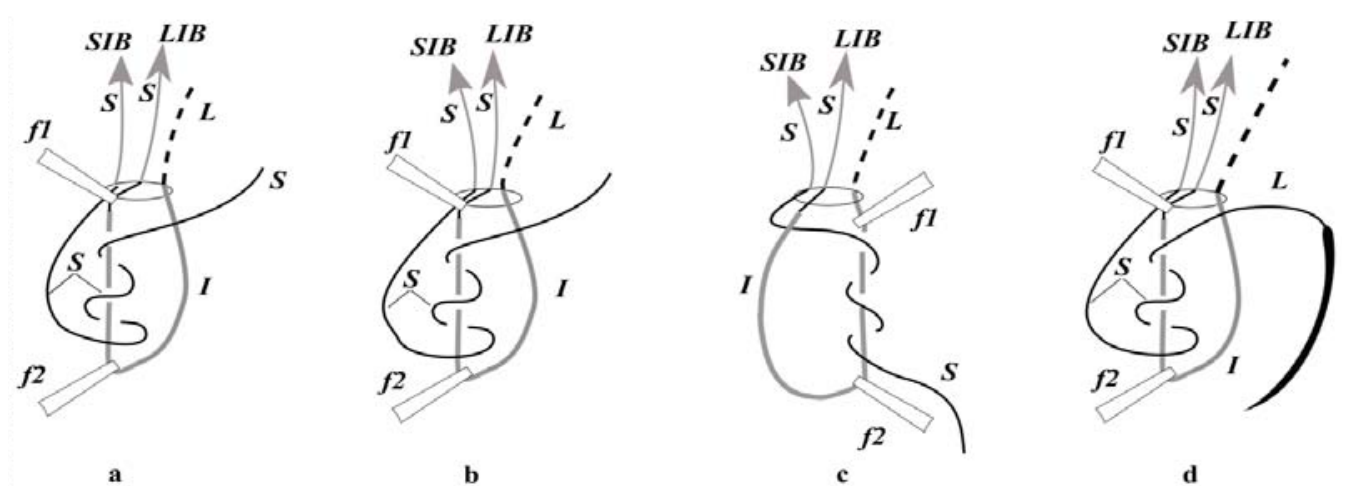

Figure 6: Technical variants of Siepser knot construction, new terms: a) Siepser [1]; b) Condon [9]; c) Ahmed [10]; d) Yao and Jhanji [11].

intimate mechanism is the same: in all variants, $\mathbf{S}$ makes throws around (I), toward LIB.

Instead of the present situation, when 6 variants of knot construction are described [1,8-12] (and God knows how many - in the future), we will have only 2 variants: one variant produces granny's or square knot by throwing $\boldsymbol{S}$ around $(\boldsymbol{I}),[1,8,12]$ and the other variant achieves the same result by coiling (I) around an instrument and by pulling $\boldsymbol{S}$ through these coils [9-11]. The new (I) is made of 1 piece, instead of being composed of 2 segments [(I) and C], so that both in Condon and in Ahmed variants, the angled forceps is held above the new (I) with the tip toward the new $L$; the coils are made in (I), and the new $\mathbf{S}$ is pulled through these coils toward $\mathbf{L I B}$, in a manner which is similar to any other Siepser-type procedure. The only difference is that one variant [9] makes the coils in (I) close to $\boldsymbol{S}$, while the other [10]- in (I) close to $L$.

With the new names and limits of $(I)$, we have succeeded to unify in theory all variants of Siepser knot, because in all variants, $\mathbf{S}$ is thrown around (I) toward $\boldsymbol{L} I \boldsymbol{B}$, no matter if $\boldsymbol{S}$ is literally thrown around (I), or coils are made in (I) and $\boldsymbol{S}$ is dragged through those coils.

\section{Discussions and Conclusions}

\section{The new names and limits of $(I)$ bring several advantages}

1) The new terminology is simple to assimilate, because it respects the reality: speaking in symbols, $\mathbf{S}$ will always be short, and $\boldsymbol{L}$ will always be long. This is an intended pleonasm, to underline the fact that for the first time, the terms are self-defining, instead of the present situation when the reality is crowded into a name that frequently does not reflect it. As consequence, the learning curve will be shortened, and the errors caused by misunderstanding will be fewer.

2) With the new terms, the differences connected with the patient's laterality, with the doctor's hand dominance, and with the surgical progress in different variants, disappear. With these terms, I repeat, errors caused by misunderstanding are less possible.

3) The new limits of filament segments ease the repartition of the main functions per filament in Siepser-type procedures: $\mathbf{S}$ will fulfill the suture purpose; (I) will participate to knot construction - together with $\boldsymbol{S}$, while $\boldsymbol{L}$ will drag the loose knot inside AC, and will tighten it - together with $\mathbf{S}$.

4) The new names and limits of (I) allow, for the first time, the establishment of rules for success in Siepser-type sutures, valid in all situations. These rules will be detailed in one of my next papers.

5) The new names and limits of (I) simplify the understanding of Siepser-type variants.

For the first time, the practitioner may use his judgment before operating, because all gestures have a logic caught in rules for success, instead of the present situation when the practitioner repeats a succession of gestures, because thus he has seen his tutor doing.

6) The new limits of (I) allow the observation of a bridge between McCannel and Siepser types of procedures, a bridge difficult to observe with the old limits of (I): with the new limits of (I), the whole filament pathway in McCannel-type procedures is reproduced by the pathway of the new $\boldsymbol{S}$ in Siepser-type ones (Figure 5). The suggested terminology and limits of (I) are valid not only for any variant of Siepser knot practiced in any particular situation imposed by local configurations or by surgeon's hand dominance, but also in any variant of McCannel procedure, in which unequal filament ends facilitate their identification and alternation in the knot construction - the essential maneuver for the creation of square knot.

7) The theoretical consequence of these facts of observation is a unifying, clear, and all-inclusive classification of all procedures for iris suture, with a common stem, 2 branches (McCannel and Siepser), and with leaves represented by technical variants.

The practical result is that if the Siepser procedure would accidentally stop after the filament gets out from $\mathbf{S P}$ (accidental filament cut, or break), we could end the suture according to McCannel procedure. Going further, in case of accidents, if only the passage through SIB and $L I B$ remained intact, with a rest of filament of around $3 \mathrm{~cm}$, we could avoid ablating the filament and repeating the iris trauma with new needle passages. With a little additional effort, the suture may continue with the remaining filament, using either McCannel or Siepser type classical variants, not the difficult intraocular knot construction and tightening according to Ahmed [17]. The paper on how to achieve this is in preparation.

8) The paper suggests the use of a new term in the domain of iris suture: the knot tightening should be performed in an isometric manner, meaning without changing the relative position of intraocular structures one in rapport with the others, "without iris stretch". 


\section{References}

1. Siepser SB. The closed chamber slipping suture technique for iris repair. Ann Ophthalmol. 1994; 26: 71-72.

2. Bordeianu CD. "Flap radiofrequency capsulorhexis in lens subluxation": in "Original procedures for lens subluxation". The $7^{\text {th }}$ Congress of the Romanian Society of Ophthalmology, Sinaia. 2008, Abstract book $p 5$.

3. Siegel MJ, Condon GP. Single suture iris-to-capsulorhexis fixation for in-thebag intraocular lens subluxation. J Cataract Refract Surg. 2015; 41: 2347 2352.

4. Bordeianu CD. Personal procedure to solve lens subluxation without suture in the sulcus. The $26^{\text {th }}$ Congress of ESCRS, Berlin. 2008, Abstract book, p. 21.

4'. Bordeianu CD. Letter to Editor: Use of filaments on CTR eyelets, to allow its insertion and manipulation in an "isometric" manner. J Cataract Refract Surg. 2021; in print.

5. Bordeianu CD. [The guiding cannula in iris suture] (Romanian). SSM lassy. 1984.

6. Bordeianu CD. A new manner of sleeveless phacoemulsification, destined to spare the relatively fragile diathermic capsulorhexis rim. The $22^{\text {nd }}$ Congress of ESCRS Paris. 2004; Abstract book, p 122.

7. Sourdille P. "Immobile movement" concept enhances safety of bimanual microincision surgery. Ocular surgery news. October $1^{\text {st }} 2007$.

8. Osher RH, Snyder ME, Cionni RJ. Modification of the Siepser slip-knot technique. J Cataract Refract Surg. 2005; 31: 1098-1100.

9. Condon GP. Siepser Sliding Knot Demo. Posted in 2009-2014. 2020.
10. Ahmed Ikbal K. Modified Siepser Sliding Knot for Iris Suturing. 2013.

11. Yao Y, Jhanji V. Simplified pupilloplasty technique through a corneal puncture to manage small iris coloboma or traumatic iris defect. AME Med J. 2017; 2 : 31.

12. Priya Narang, Amar Agarwal. Single-pass four-throw technique a new option for pupiloplasty. Healio Ophthalmology. January, 2017.

13. Schoenberg ED, Price FW Jr. Modification of Siepser sliding suture technique for iris repair and endothelial keratoplasty. J Cataract Refract Surg. 2014; 40 : 705-708.

14. Dunn SP, Stec Lori. Iris reconstruction: in Ophthalmic Microsurgical Suturing Techniques. MS Macsai, Springer-Verlag, Berlin, Heidelberg, 2007: 71-83.

15. Gulden Ophthalmics. Siepser Sliding Knot by Gulden Ophthalmics. 2011.

16. Eun Hye Jung, Mee Kum Kim, Won Ryang Wee. In Situ Peripheral Iridoplasty in Phakic Eyes for the Treatment of Symptomatic Peripheral Iridotomy. Korean J Ophthalmol. 2014; 28: 426-428.

17. Singh Harmanjit, Ahmed Iqbal K. Techniques for Iris Repair and Pupilloplasty. 2014.

18. Condon GP. The Siepser Sliding Knot. CRST Europe. Mar, 2014.

19. Brandon Ayres. Sliding knot model. 2014.

20. Ahmed Ike K, Patrick Gooi. Pupilloplasty and Suturing Iris Defects. Posted june 3,2020

21. McCannel M. A retrievable suture idea for anterior uveal problems. Ophthalmic Surgery. 1976; 7: 98-103. 